\title{
EVALUATION OF STATIC STRENGTH OF WELDED DISK OF SMOKE EXHAUSTER IMPELLER
}

\author{
A.V. MOLTASOV ${ }^{1}$, P.N. TKACH ${ }^{1}$, A.Ya. GOGOLEV ${ }^{2}$, A.A. AVDYUSHKIN ${ }^{2}$ and S.I. MOTRUNICH ${ }^{1}$ \\ ${ }^{1}$ E.O. Paton Electric Welding Institute, NASU \\ 11 Kazimir Malevich Str., 03680, Kiev, Ukraine. E-mail: office@paton.kiev.ua \\ ${ }^{2} \ll$ Soyuzenergomash» LLC \\ 8 Gavanskaya Str., 49127, Dnepr, Ukraine. E-mail:recept_prmz@souzenergo.com
}

\begin{abstract}
A checking calculation of static strength was carried out for welded structure of a disk of smoke exhauster impeller under service loading. The impeller consists of the disk and welded to it cylinder shell with twenty blades uniformly located along its perimeter. Stressed state, being realized in the disk during operation, is caused by effect of inertia appearing as a result of impeller rotation with a constant angular velocity. The stress components were determined by solving a boundary problem of quasi-static elasticity theory. Setting of the boundary conditions required to determine stress on a disk outer profile, promoted by centrifugal forces, developed by blades. A corresponding formula was proposed for this taking into account mass-centering characteristics of the blade. It is determined that the maximum radial stress of the stamped disks of 48 and $44 \mathrm{~mm}$ thickness acts in a hub and makes 28 and $29.5 \mathrm{MPa}$, respectively. The maximum circumferential stresses at that are more than two times lower than the radial ones. The main difference between welded and stamped disks is a presence of stress concentrators, caused by geometry inhomogeneity of structure in zone of welded joints. Stressed state in the zone of stress concentration was described using an engineering method based on broken-sections hypothesis. The results of calculations of a stress concentration factor by proposed method well agree with the results, received by a finite element method. Taking into account stress concentration in the welded disks of 48 and $44 \mathrm{~mm}$ thickness the maximum radial stress acts in the place of ring to shell conjugation and makes 35.8 and $37.5 \mathrm{MPa}$, respectively. These values exceed stresses in the hub for corresponding stamped disks more than by $20 \%$, however it is 5 times lower than the yield strength of disk material. It can be state due to this that static strength is provided for both studied welded structures of the disk. 14 Ref., 12 Figures.
\end{abstract}

Keywords : smoke exhauster impeller, welded disk, service loads, checking calculation, stressed state, stress concentration, broken-sections hypothesis

Ensuring a continuous operation of heat power plants (HPP) is an important strategic direction for Ukrainian power engineering under modern economic conditions. Stable operation can be provided only under timely planned preventive repairs (PPR), which include replacement of the parts and assemblies of power installations. At that it is a significant dependence of domestic enterprises on the foreign components for these installations, to which the impellers of HPP smoke exhausters are referred. In order to reduce import dependence and preserve PPR terms it is necessary to develop domestic design being as good as imported on strength, technological effectiveness and service properties. At that it should be reasonable from economic point of view. The imported analogue applies an impeller design with stamped disks of $1940 \mathrm{~mm}$ diameter. Manufacture of the stamps of such a diameter is not reasonable from economic point of view. Therefore, «Soyuzenergomash» LLC developed the variants of disk welded structure, two of which (Figure 1) were delivered to the E.O. Paton Electric Welding Institute for evaluation of their working capacity. Grounding one of the variants requires determination of stress-strain state (SSS) of the disks taking into account stress concentration caused by their geometry in welds zone.
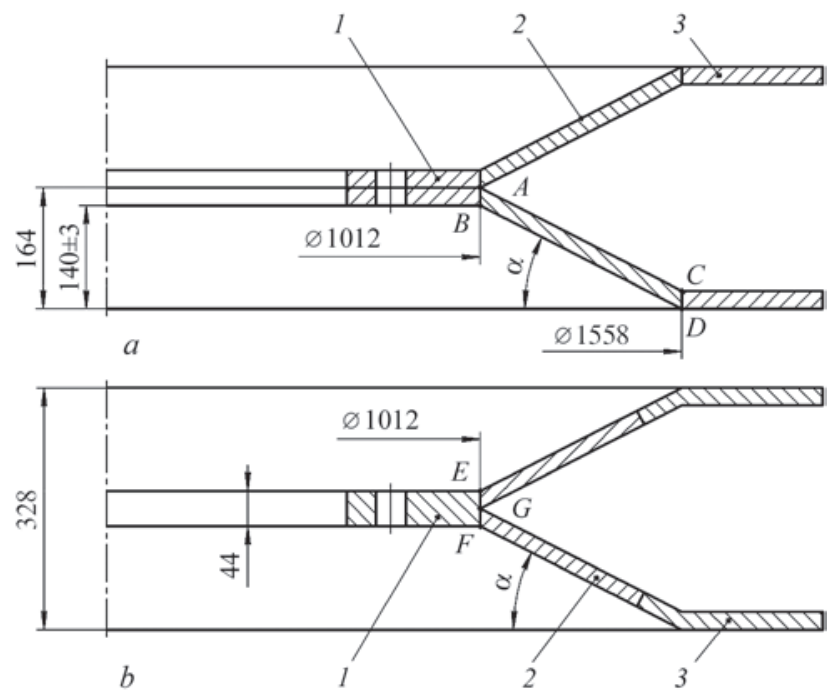

Figure 1. The first ( $a$ ) and second $(b)$ variants of performance of welded disk of smoke exhauster impeller: 1 - ring; 2 - shell; 3 - edge 


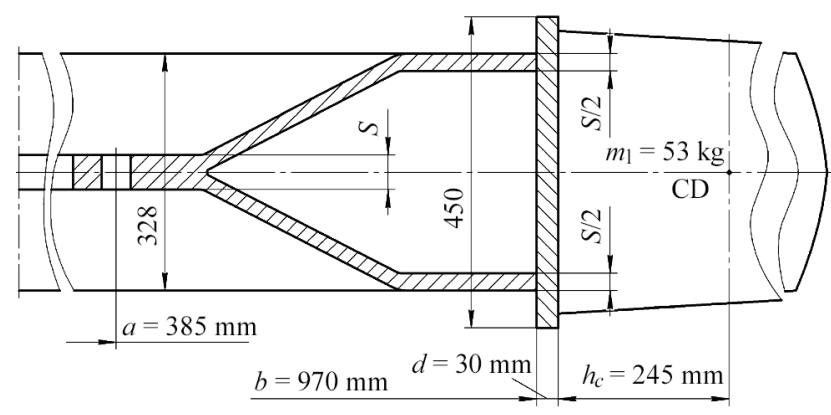

Figure 2. Impeller calculation scheme considering blades

At $a$ distance the impeller disk is rigidly fixed by means of bolted joint and at distance $b$ it contains a welded cylinder shell with twenty blades, uniformly located along its perimeter (Figure 2).

During operation the smoke exhauster impeller rotates at a constant angular velocity

$$
\omega=\frac{\pi n}{30},
$$

where $n$ is the impeller rotation frequency, rev/min.

Rotation in a blade root section promotes for appearance of centrifugal force [1]

$$
P_{c}=m r_{c} \omega^{2},
$$

where $m$ is the blade mass; $r_{c}$ is the distance from rotation axis to blade mass center, determined by formula

$$
r_{c}=b+d+h_{c},
$$

where $d$ is the thickness of cylinder shell; $h_{c}$ is the blade mass center, which for truncated pyramid (Figure 3 ) is determined by formula [2]

$$
h_{c}=\frac{H}{4} \frac{F_{1}+2 \sqrt{F_{1} F_{2}}+3 F_{2}}{F_{1}+\sqrt{F_{1} F_{2}}+F_{2}} .
$$

If it is assumed that the centrifugal forces, developed by the blades, are uniformly distributed over the impeller surface (Figure 4), then stress on its outer radius considering (2) can be determined from formula

$$
p_{c}=\frac{z m r_{c} \omega^{2}}{2 \pi b s}
$$

where $z$ is the number of blades; $s$ is the disk thickness.

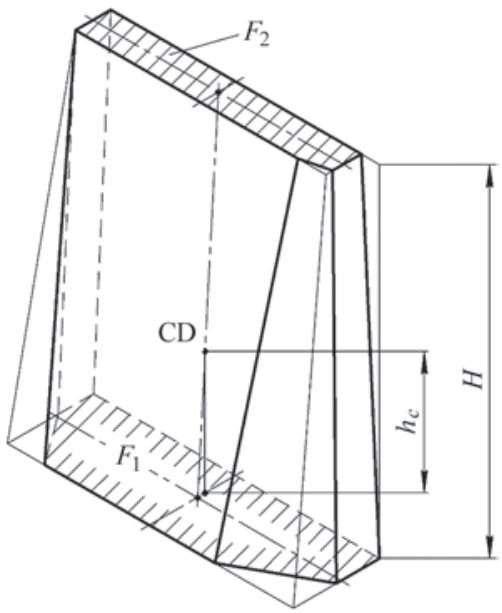

Figure 3. Blade model for mass center determination
Regardless the developed geometry, thickness of the disk $s$ in radial section is constant along the radius. Due to small $s / b$ relationship in the considered case a plane stressed state is formed in the impeller and stresses can be considered constant on thickness [3].

This problem can be solved as a quasi-static problem of elasticity theory, if according to d'Alembert's principle the impeller is stopped and corresponding volume forces are applied to it [4].

Solution of the problem about rotating disks is well known in scientific-and-technical literature $[5,6]$, therefore, without mentioning mathematical computations, we bring an expression for radial movement $u$ as well as radial $\sigma_{r}$ and circumferential $\sigma_{\theta}$ stresses, appearing in the disk.

$$
\begin{gathered}
u=\frac{1-\mu^{2}}{E}\left(C_{1} r+C_{2} \frac{1}{r}-\frac{\rho \omega^{2}}{8} r^{3}\right), \\
\left\{\begin{array}{l}
\sigma_{r}=(1+\mu) C_{1}-(1-\mu) C_{2} \frac{1}{r^{2}}-(3+\mu) \frac{\rho \omega^{2}}{8} r^{2} ; \\
\sigma_{\theta}=(1+\mu) C_{1}+(1-\mu) C_{2} \frac{1}{r^{2}}-(1+3 \mu) \frac{\rho \omega^{2}}{8} r^{2},
\end{array}\right.
\end{gathered}
$$

where $\mu$ is the coefficient of lateral deformation; $\rho$ is the density of disk and blade material; $C_{1}, C_{2}$ are the constants of integration, determined from boundary conditions on the inner and outer edge.

Since the impeller is rigidly fixed on inner radius and no movements there are possible $u=0$ at $r=$ $=a$. Radial stresses $p_{c}$, developed by blade centrifugal forces, act on outer radius, therefore, $\sigma_{r}=p_{c}$ at $r=b$. A system of equations is received by inserting the boundary conditions in equation (6) and the first from equations (7). Its solution provides us with

$$
\begin{aligned}
& C_{1}=\frac{p_{c} b^{2}+\frac{\rho \omega^{2}}{8}\left[(3+\mu) b^{4}+(1-\mu) a^{4}\right]}{(1+\mu) b^{2}+(1-\mu) a^{2}} ; \\
& C_{2}=-a^{2} b^{2} \frac{p_{c}+\frac{\rho \omega^{2}}{8}\left[(3+\mu) b^{2}-(1+\mu) a^{2}\right]}{(1+\mu) b^{2}+(1-\mu) a^{2}} .
\end{aligned}
$$

Knowing an area of larger and smaller blade roots $F_{1}=16440 \mathrm{~mm}^{2}$ and $F_{2}=6333 \mathrm{~mm}^{2}$, respectively, and its height $H=578 \mathrm{~mm}$, let's determining using formula (4) a distance from the root section to blade mass center $h_{c}=245 \mathrm{~mm}$.



Figure 4. Scheme of loading of smoke exhauster impeller

ISSN 0957-798X THE PATON WELDING JOURNAL, No. 12, 2016 
Considering that outer diameter of the disk $b=970 \mathrm{~mm}$, and thickness of the cylinder shell $d=$ $30 \mathrm{~mm}$, it is possible to determine the distance from rotation axis to blade mass center $r_{c}=1245 \mathrm{~mm}$.

At set number of blades $z=20$ and rotation velocity of the disk $n=500 \mathrm{rev} / \mathrm{min}$, assuming that a calculated mass of one blade is $m=53 \mathrm{~kg}$ and after preliminary calculation by formula (1) $\omega=52.3 \mathrm{rad} / \mathrm{s}$, let's determine using formula (5) a value of radial stress, acting on disk outer profile:

- for the first variant (at $s=48 \mathrm{~mm}$ ) $p_{c}=13.6 \mathrm{MPa}$;

- for the second variant (at $s=44 \mathrm{~mm}$ ) $p_{c}=14.8 \mathrm{MPa}$.

The curves of radial and circumferential stresses along the radius (Figure 5) are plotted by formula (7) knowing disk inner diameter $a=385 \mathrm{~mm}$, and taking material density for steel disk $\rho=7800 \mathrm{~kg} / \mathrm{m}^{3}$ and coefficient of lateral deformation $\mu=0.3$.

It follows from stress distribution that the largest stress acts in radial direction in the place of impeller to hub fixing and makes for the first and second variants 28 and 29.5 MPa, respectively. At that, the value of maximum radial stress in both cases is more than two times exceeds the value of maximum circumferential stress.

Since residual welding stresses were removed by means of respective heat treatment, the most significant difference of the disk welded structure from stamped variant is appearance in the weld zones of stress concentration, promoted by transfer from one part being joined to another, and value of acting radial stress will be determined by formula

$$
\sigma_{r}=\alpha_{\sigma} \sigma_{r}^{\text {nom }},
$$

where $\sigma_{r}^{\text {nom }}$ is the radial stress without concentration, determined by first formula (7); $\alpha_{\sigma}$ is the stress concentration factor (SCF).

For the first variant let's consider concentration of stresses in a zone of joining shell 2 to ring 1 (points $A$ and $B$ ) and in zone of joining shell 2 to edge 3 (points $C$ and $D$ ) (see Figure 1, $a$ ). For the second variant the concentration should be taken into account only for a weld joining shell 2 to ring 1 (points $E$ and $F$ ) (see Figure $1, b)$, since weld reinforcement, joining shell 2 to edge 3 , is removed. The weld zone (point $G$ ) provides for smooth transfer between structure elements being joined (see Figure 1,b), therefore, it can be taken out from consideration, since stress concentration factor is rapidly reduced with curvature decrease [7].

An engineering method, based on broken sections hypothesis [8, 9], is used for investigation of stressed state in the zone of stress concentration. It guarantees high accuracy for different types of welded joints at various variants of loading $[10,11]$.

Pass a plane-broken section $A B C D G H$ (Figure 6). The end areas of section $A B$ and $G H$ are normal to profile, length of these areas $a_{0}$ characterizes the depth of concentrator action


Figure 5. Distribution of radial $\sigma_{r}$ and circumferential $\sigma_{\theta}$ stresses on radial coordinate in disk of the first $(a)$ and second $(b)$ variants

$$
a_{0}=2 \sqrt{t R}
$$

where $t$ is the height of curving.

The middle areas of section $B C$ and $D G$, which are regulated by hypothesis of plane sections, are passed perpendicular to applied load.

Pass the second plane-broken-section $A_{1} B_{1} C_{1} D_{1} G_{1} H_{1}$ through point $A_{1}$, located at small distance from point $A$. It is assumed by convention that $A_{1} B_{1} C_{1} D_{1} G_{1} H_{1}$ section remains stable in deformation, and the section $A B C D G H$ takes a position marked by dashed line. At that $K F$ fiber, situated at distance $u$ from the axis and located normal to area $A B$, receives $K N$ elongation.

Relative elongation of $K F$ fiber

$$
\varepsilon_{u}=\frac{K N}{K F}=\frac{K L \cos \beta}{(R+s / 2-u) \Delta \beta} .
$$

Normal stress in this fiber according to Hooke's law

$$
\sigma_{u}=\frac{K L \cos \beta}{(R+s / 2-u) \Delta \beta} E .
$$

Now let's consider fiber $Q S$, situated at a distance $v$ from the axis and located normal to $B C$ area. As a result of deformation this fiber will have $S T$ elongation and normal stress will appear in it

$$
\sigma=\frac{S T}{Q S} E=\frac{K L}{(R+a) \Delta \beta \cos \beta} E
$$

Equilibrium condition is as follows

$$
p s=2 \int_{s / 2-a_{0}}^{s / 2} \sigma_{u} d u \cos \beta+2 \int_{y_{C}}^{y_{B}} \sigma_{v} d v,
$$

where

$$
\begin{gathered}
y_{B}=\frac{s}{2}+R(1-\cos \beta)-a_{0} \cos \beta ; \\
y_{C}=\left(\frac{s}{2}+R\right)(1-\cos \beta) .
\end{gathered}
$$




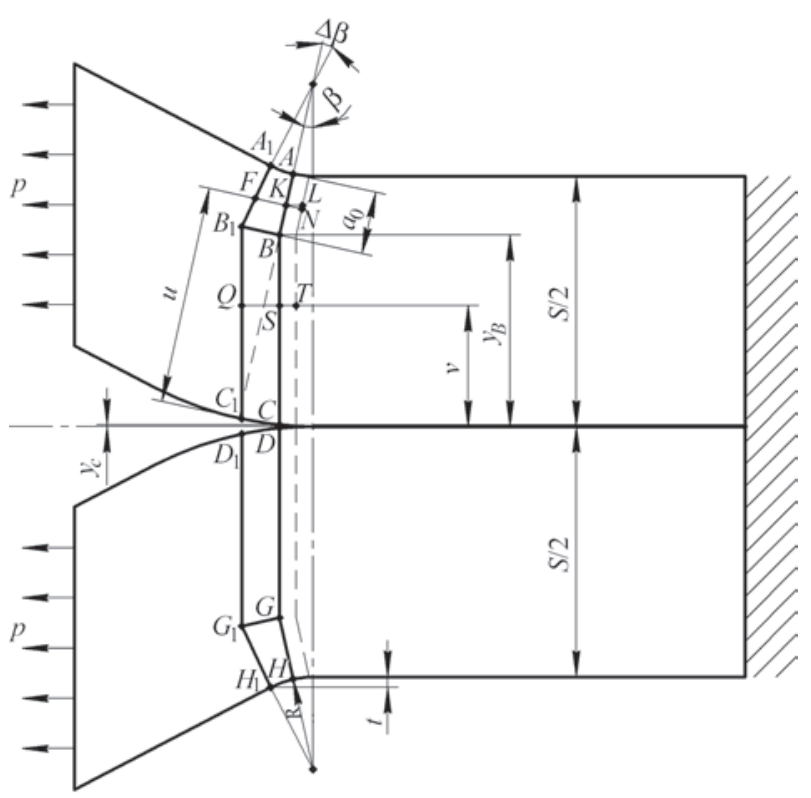

Figure 6. Construction of plane-broken sections in stress concentration zone

The next is received after integration by inserting $\sigma_{u}$ from (10) and $\sigma_{v}$ from (11) in the equilibrium condition (12)

$$
\frac{K L E}{\Delta \beta}=\frac{p s}{2 k},
$$

where $k$ is the geometry characteristic of section

$$
k=\ln \left(1+\frac{a_{0}}{R}\right) \cos ^{2} \beta+\frac{s / 2-a_{0}}{R+a_{0}} .
$$

Inserting expression (13) in the dependencies (10) and (11) the expressions for stresses in areas $A B$ and GH are obtained

$$
\sigma_{u}=\frac{p s \cos \beta}{2 k(R+s / 2-u)}
$$

and in areas $B C$ and $D G$

$$
\sigma_{v}=\frac{p s}{2 k\left(R+a_{0}\right) \cos \beta}
$$

respectively.

Analysis of expressions (14) and (15) shows that stress is constant on thickness in areas $B C$ and $D G$ and varies only from section to section. In areas $A B$ and $G H$ stress is changed on hyperbolic law and reaches the maximum on a surface in points $A$ and $H$, i.e. at $u=\mathrm{s} / 2$

$$
\sigma_{u}^{A}=\frac{p s \cos \beta}{2 k R},
$$

respectively $S C F$ is determined by formula

$$
\alpha_{\sigma}=\frac{\sigma_{u}^{A}}{p}=\frac{s \cos \beta}{2 R\left[\ln \left(1+\frac{a_{0}}{R}\right) \cos ^{2} \beta+\frac{s / 2-a_{0}}{R+a_{0}}\right]} .
$$

In calculation of $S C F$ for the second variant of impeller design in (12) it is necessary to assume $y_{C}=0$, then

$$
=\frac{\alpha_{\sigma}=}{2 R\left[\ln \left(1+\frac{a_{0}}{R}\right) \cos ^{2} \beta+\frac{s / 2+R(1-\cos \beta)-a_{0} \cos \beta}{\left(R+a_{0}\right) \cos \beta}\right]} .
$$

Investigation of functions (16) and (17) on procedure [12] showed that $\alpha_{\sigma}$ monotonously decrease at rise of $\beta$ in the interval

$$
\beta \in[0 ; \alpha],
$$

where

$$
\alpha=\arccos \left(1-\frac{t}{R}\right)
$$

therefore, it can be stated, that for two variants the $S C F$ structure has the maximum value at $\beta=0$.

As it was mentioned earlier the main geometry parameter affecting $S C F$ value is a curvature, therefore it is necessary to determine conjugation radius $R$ in the weld zones (points $B$ and $C$ for the first variant and points $E$ and $F$ for the second).

Butt weld S25 is used in assembly of the disk on variant I in accordance with GOST 5264-80, which provides for double groove preparation with $\gamma=$ $25 \pm 2^{\circ}$ and gap $\delta=0-3 \mathrm{~mm}$ at $24 \mathrm{~mm}$ metal thickness. Shell angle in relation to rotation plane can be determined from relationship of sizes, marked on drawing (Figure 1,a). Since one of the sizes is set with tolerance, then angle $\alpha$ will be varied in $26.65-27.65^{\circ}$ range. It is expected that conjugation radius $R$ will be minimum, i.e. corresponding to the largest SCF value at maximum values of angle $\alpha$. Since in practice the limiting values of sizes are unlikely, $\alpha$ close to the maximum angle $27.5^{\circ}$ is taken.

Since the weld will be made at inclined relative position of the parts (Figure 7,a), then reinforcement removal with smooth transfer can be performed on conjugation radiuses in points $B$ and $C$. Actual parameters of groove preparation will effect their value, moreover the smallest conjugation radiuses are received in choosing the dimensions on low limiting deviation $\gamma=23^{\circ}, \delta=0$.

The conjugation radiuses $r_{B}$ and $r_{C}$ can be determined from known relationship between the radius and chord at known central angle [2]

$$
r_{B(C)}=\frac{a_{B(C)}}{2 \sin \frac{\alpha}{2}}=\frac{S}{2}\left(\operatorname{tg} \gamma \operatorname{ctg} \frac{\alpha}{2}-1\right) .
$$

It is obvious that $r_{B(C)}(\alpha)$ monotonously decreases in $\alpha=26.65-27.65^{\circ}$ interval, that proves the necessity of selection of the largest radius $\alpha$, since at that the received radiuses of conjugation $r_{B}$ and $r_{C}$ are minimum.

Two shells are welded up to a ring using non-standard weld at $\alpha=27 \pm 1^{\circ}$ angle in disk assembly on variant II.

A calculation of stressed state of the structure second variant is also reasonably to carry out for the 

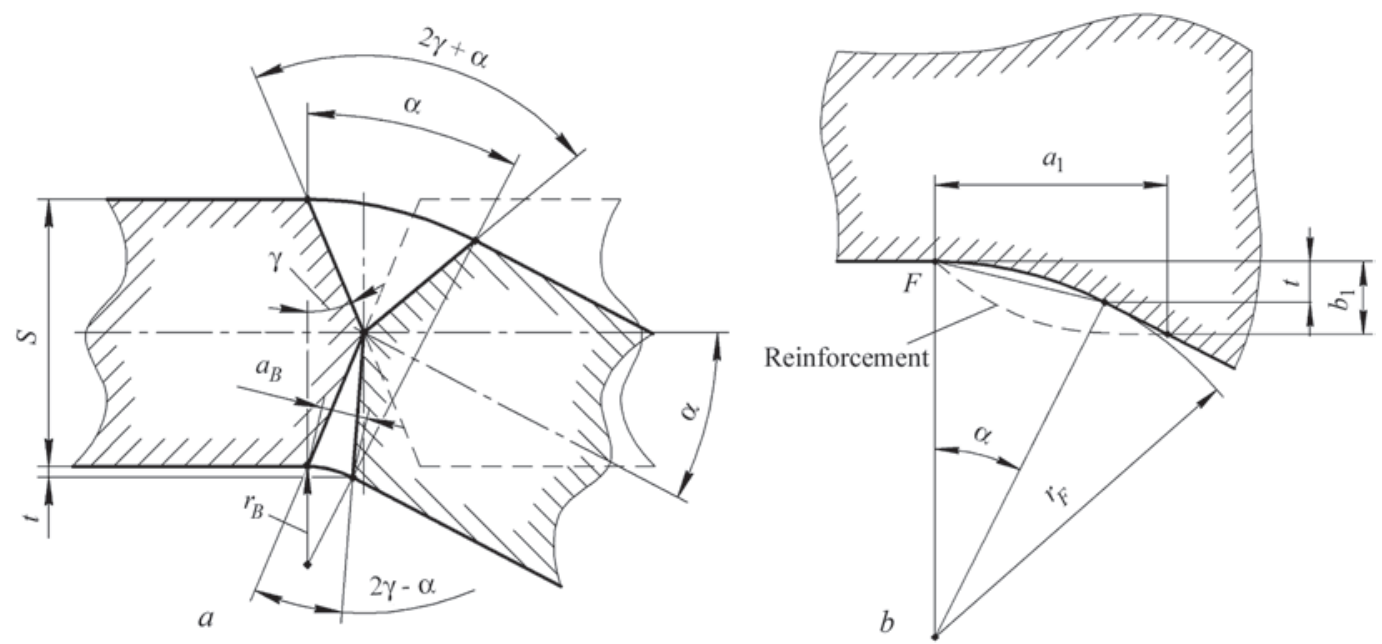

Figure 7. Determination of conjugation radiuses of surfaces of parts for the first $(a)$ and second $(b)$ variants

worst combination of tolerances. For this it is necessary to obtain the dependence of radiuses of conjugation $r_{E}$ and $r_{F}$ on the main parameters $a$ and $b$ of the weld (Figure 7, $b$ ).

$$
r_{E}=r_{F}=\frac{a_{1} \operatorname{tg} \alpha-b_{1}}{\sin \alpha \operatorname{tg} \alpha-2 \sin ^{2} \frac{\alpha}{2}} .
$$

Analysis of dependence (18) shows that in $\alpha=26$ $28^{\circ}$ range the minimum radius corresponds to the minimum value of $a_{1}$ and the maximum value of $b_{1}$. $r_{E(F)}(\alpha)$ function monotonously rises in the researched interval, therefore, the minimum radiuses $r_{E}$ and $r_{F}$ correspond to the minimum value of $\alpha$.

Inserting in formula (19) assumed values $\gamma=23^{\circ}$ and $\alpha=27.5^{\circ}$, let's determine $r_{B}=r_{C}=8.82 \mathrm{~mm}$, and assuming $R=r_{B}=r_{C}$ in formula (18), $t=1 \mathrm{~mm}$ is determined.

Inserting in formula (20) $a_{1}=14 \mathrm{~mm}, b_{1}=6 \mathrm{~mm}$ and $\alpha=26^{\circ}$ values, the smallest conjugation radiuses $r_{E}=r_{F}=7.36 \mathrm{~mm}$ are received and, assuming $R=r_{E}=$ $=r_{F}$ in formula (18), $t=0.74 \mathrm{~mm}$ is determined.

Inserting in formula (9) $R=r_{B}=r_{C}=8.82 \mathrm{~mm}$ and $t=1 \mathrm{~mm}$, let's determine $a_{0}=5.94 \mathrm{~mm}$, then according to formula (16) at $\beta=0^{\circ} \mathrm{RCF}$ takes $\alpha_{\sigma}=1.56$ value, and at $\beta=\alpha=27.5^{\circ} \mathrm{RCF}$ takes its minimum value $\alpha_{\sigma}=1.44$.

Inserting in formula (9) $R=r_{B}=r_{C}=7.36 \mathrm{~mm}$ and $t=0.74 \mathrm{~mm}$, let's determine $a_{0}=4.67 \mathrm{~mm}$, then according to formula (17) at $\beta=0^{\circ}$ SCF takes $\alpha_{\sigma}=1.55$ value, and at $\beta=\alpha=26^{\circ}$ SCF takes its minimum value $\alpha_{\sigma}=1.25$.

Computer modelling of stressed state in the zones of stress concentration (Figures 8 and 9) was carried out for validation of the obtained formulae (16) and (17).

Elastic axial tension problem was solved in simulation for steel with physical-chemical properties of steel 20 at $200{ }^{\circ} \mathrm{C}$ : modulus of normal elasticity $E=1.9 \times 10^{5} \mathrm{MPa}$, Poisson's coefficient $\mu=0.3$. The problem was solved for two-dimensional model, representing itself an element of impeller sector of unit thickness. A limitation in form of rigid fixing was applied from hub side, and unit load, distributed on the edge, (Figure 8) was from disk side.

It was determined based on results of the numerical calculation (Figure 9) that the maximum values of SCF for the first and second variants made 1.539 and 1.533, respectively that well agree with the results received using mentioned above calculation method.

From constructions (Figure 10, $a$ ) it is determined that $\beta=0^{\circ}$ value corresponds to $r=500.91 \mathrm{~mm}$ and $r=$ $784.09 \mathrm{~mm}$ values, and $\beta=\alpha=27.5^{\circ}$ corresponds to $r=$ $507.02 \mathrm{~mm}$ and $r=777.98 \mathrm{~mm}$ values, thus we can show a curve of radial stresses in the disk of the first variant taking into account stress concentration (Figure 11, a).

From constructions (Figure 10, $b$ ) it is determined that $\beta=0^{\circ}$ value corresponds to $r=502.78 \mathrm{~mm}$ value, and $\beta=\alpha=26^{\circ}$ corresponds to $r=506 \mathrm{~mm}$ value,
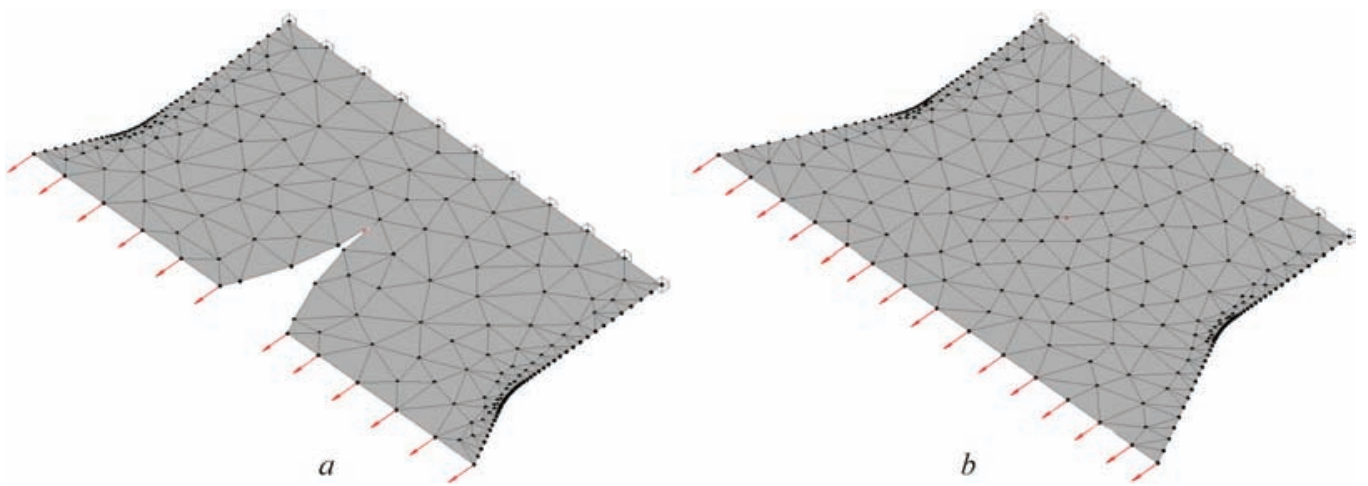

Figure 8. Conditions of loading, fixing and finite element breaking up of fragments of disks of the first $(a)$ and second $(b)$ variants 




Figure 9. Stress fields in concentration zones of disks of the first $(a)$ and second $(b)$ variants at axial tension by unit load
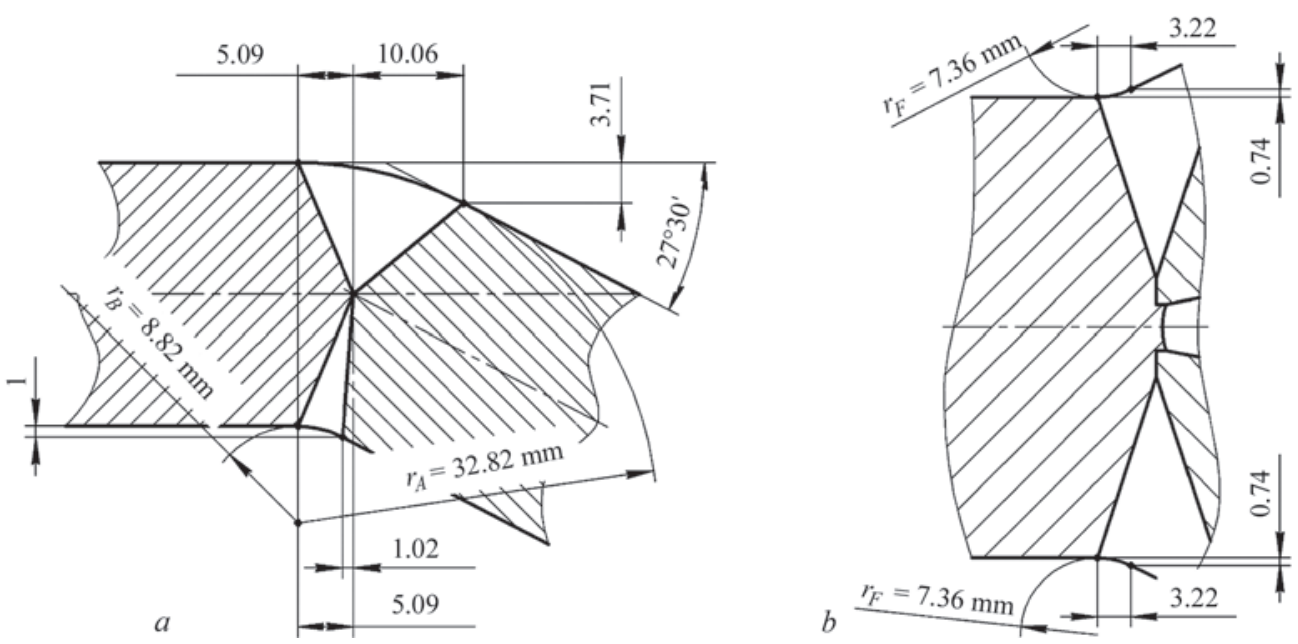

Figure 10. Dimensions in conjugation zone for the first $(a)$ and second $(b)$ variants of performance of welded disk of smoke exhauster impeller


Figure 11. Distribution of radial stresses in disk of the first $(a)$ and second $(b)$ variants considering stress concentration

thus we can show a curve of radial stresses in the disk of the second variant taking into account stress concentration (Figure 11, b).

The curves of radial stresses considering concentration in the weld zone show that the maximum radi- al stress in the welded disk of the first variant acts in $r=500.91 \mathrm{~mm}$ point and makes $35.8 \mathrm{MPa}$, and in the disk of the second variant it is in the point $r=502.78 \mathrm{~mm}$ and makes $37.5 \mathrm{MPa}$. These values for both variants of 


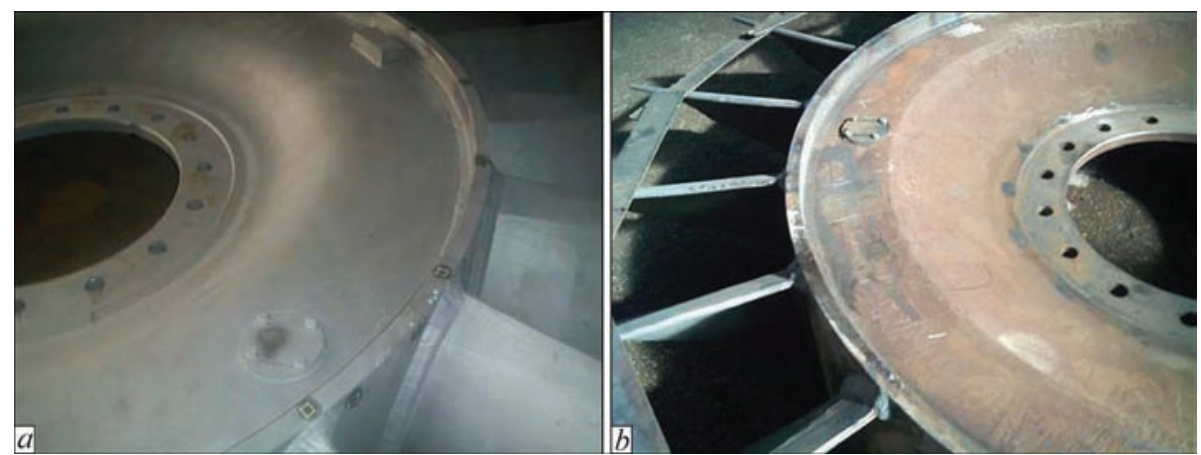

Figure 12. Smoke exhauster impeller with stamped $(a)$ and welded $(b)$ disk

the welded disk is more than $20 \%$ exceed the value of stress in the hub for corresponding stamped disks.

Analysis of stressed state shows that the maximum stresses in the disks of both variants make not less than $0.2 \sigma_{\mathrm{y}}$ (yield strength makes $230 \mathrm{MPa}$ [13] at working temperature $200{ }^{\circ} \mathrm{C}$ for steel 20 disk material). Regardless the fact that the maximum stress in the disk of the second variant is much more higher, than in the disk of the first variant, the second variant seems to be more preferable, since possible appearance of cyclic loading in the disk of the first variant provides for the probability of nucleation of fatigue cracks in two zones of stress concentration [14].

Replacement of the stamped structure by recommended variant of the welded disk was virtually realized (Figure 12).

After static and dynamic balancing, the exhauster impeller with the welded disk was put into operation at Ladyzhinsky HPP PJSC «DTEK Zapadenergo».

\section{Conclusions}

1. High-efficiency, technological and cost-effective method was developed at «Souyuzenergomash» LLC in scope of program on replacement of foreign component parts by domestic analogues for manufacture of the welded disks of smoke exhauster impellers and two variants of design of these disks structure were proposed.

2. Stuff members of the department of welded structure strength of the E.O. Paton Electric Welding Institute of the NAS of Ukraine developed the algorithm for calculation of disk static strength. It includes determination of service loads, solution of problem on elastic equilibrium of rotating disk as well as evaluation of effect of geometry inhomogeneity in the weld zone on stress distribution.

3. Calculation of stressed state for two variants of the welded disk structure was carried out. As a result, it is determined that the maximum stresses, acting in the welded disks are more than $20 \%$ higher than in the similar stamped parts, however, their value is 5 times lower the yield strength of the disk material at operating temperature.
4. Based on working conditions of the studied parts it is recommended to use a disk structure with lower amount of local stress concentrators, regardless the fact that acting in it maximum stress is somewhat higher, than in alternative design variant.

1. Kishalov, A.E., Kudoyarova, V.M., Markina, K.V. et al. (2012) Analysis of loads applied to elements of gas-turbine engine structure. Molodoj Uchyonyj, Vol. 1, 46 (11), 52-60.

2. Ryvkin, A.A., Ryvkin, A.Z., Khrenov, L.S. (1987) Mathematics handbook. Moscow: Vysshaya Shkola.

3. Timoshenko, S.P., Gudier, J. (1975) Theory of elasticity. Moscow: Nauka.

4. Babenko, A.E., Boronko, O.O., Kovalchuk, B.I. et al. (2010) Procedural guidelines for fulfillment of course and design-graphic works on the subject: Strength of materials (problems and calculation examples) for students of engineering specialities for all forms of education. Kyiv: Politekhnika.

5. Mozharovsky, M.S. (2002) Theory of elasticity, plasticity and creep: Manual. Kyiv: Vyshcha Shkola.

6. Demyanushko, I.V., Birger, I.A. (1978) Strength calculation of rotating disks. Moscow: Mashinostroenie.

7. Neuber, H. (2001) Kerbspannungslehre: Theorie der Spannungskonzentration Genaue Berechnung der Festigkeit. Vierte Verlag-Berlin: Springer-Verlag Berlin Heidelberg.

8. Verkhovsky, A.V. (1947) Hypothesis of broken sections and its application to calculation of complex configuration rods. Izvestiya TPI, 61(1), 3-46.

9. Verkhovsky, A.V., Andronov, V.P., Ionov, V.A. et al. (1958) Determination of stresses in dangerous sections of complex shape parts. Method of nonplanar sections. Moscow: Mashgiz.

10. Moltasov, A.V. (2013) Application of nonplanar section method for determination of stresses in zones of concentration caused by reinforcement of butt welded joint. Problemy Prochnosti, 1, 159-167.

11. Moltasov, A.V., Klochkov, I.N., Knysh, V.V. (2013) Engineering method of calculation of stress concentration factor in lap welded joint under extension and bending. Visnyk NTUU KPI. Seriya Mashynobuduvannya, Issue 69, 150-157.

12. Bugrov, Ya.S., Nikolsky, S.M. (2004) Higher mathematics: Manual for institutions of higher education. Vol. 2: Differential and integral calculus. Ed. by V.A. Sadovnichy. Moscow: Drofa.

13. Sorokin, V.G., Volosnikova, A.V., Vyatkin, S.A. et al. (1989) Grades of steels and alloys. Ed. by V.G. Sorokin. Moscow: Mashinostroenie.

14. Trufyakov, V.I., Dvoretsky, V.I., Mikheev, P.P. et al. (1990) Strength of welded joints under alternating loads. Ed. by V.I. Trufyakov. Kiev: Naukova Dumka. 